\title{
A Method for the Selection of Software Testing Automation Framework using Analytic Hierarchy Process
}

\author{
Mohd Sadiq \\ Software Engineering Laboratory, Lab. No. 305, \\ Computer Engineering Section, University \\ Polytechnic, Faculty of Engineering and Technology, \\ Jamia Millia Islamia (A Central University), \\ New Delhi-110025, India
}

\begin{abstract}
Software test automation framework (STAF) is a set of concepts, assumptions or practices that provide support for automated software testing. STAF includes the following for capturing and controlling the test activity, i.e., test objects, library files and reusable scripts etc. In literature, we have identified different types of automation framework like modular framework (MF), data driven framework (DDF), keyword driven framework (KDF), and hybrid framework (HF); and selecting one of them is not an easy task according to the requirements of software testing process because each framework pursues a specific objective or goal. Therefore, in order to address this issue, we present a method for the selection of software testing automation framework (STAF) using Analytic Hierarchy Process (AHP) by considering the following criteria, i.e., Master Test Script (MTS), Reading of Data from Data Files (RDDF), Data Tables and Keyword (DTK), and Scripts, Data Table and Keywords (SDTK). Finally, the utilization of the proposed approach is demonstrated with the help of an example.
\end{abstract}

\section{General Terms}

Software Testing and Analytic Hierarchy Process

\section{Keywords}

Automation Framework, Decision Making Process, AHP.

\section{INTRODUCTION}

Software testing is the process of evaluating the software behavior to check whether it operates as expected in order to improve its quality and reliability $[9,18]$. Test automation is a kind of development activity. It refers to anything that streamlines the testing process and facilitates things to move along more quickly and with less delay [2]. Test automation involves automating a manual process already in place that uses formalize testing process. Test automation involves three phases, i.e. Test planning, test reparation, and test execution [13]. Fig. 1 presents the different phases of STAF. In test planning phase following are documented: test strategy, scope of testing, concepts and the practices to be followed.

\author{
Fahamida Firoze \\ M. Tech. Scholar, Department of Computer Science \\ and Engineering, Faculty of Engineering and \\ Technology, Al-Falah University, Dhuj, Faridabad, \\ Haryana, India
}

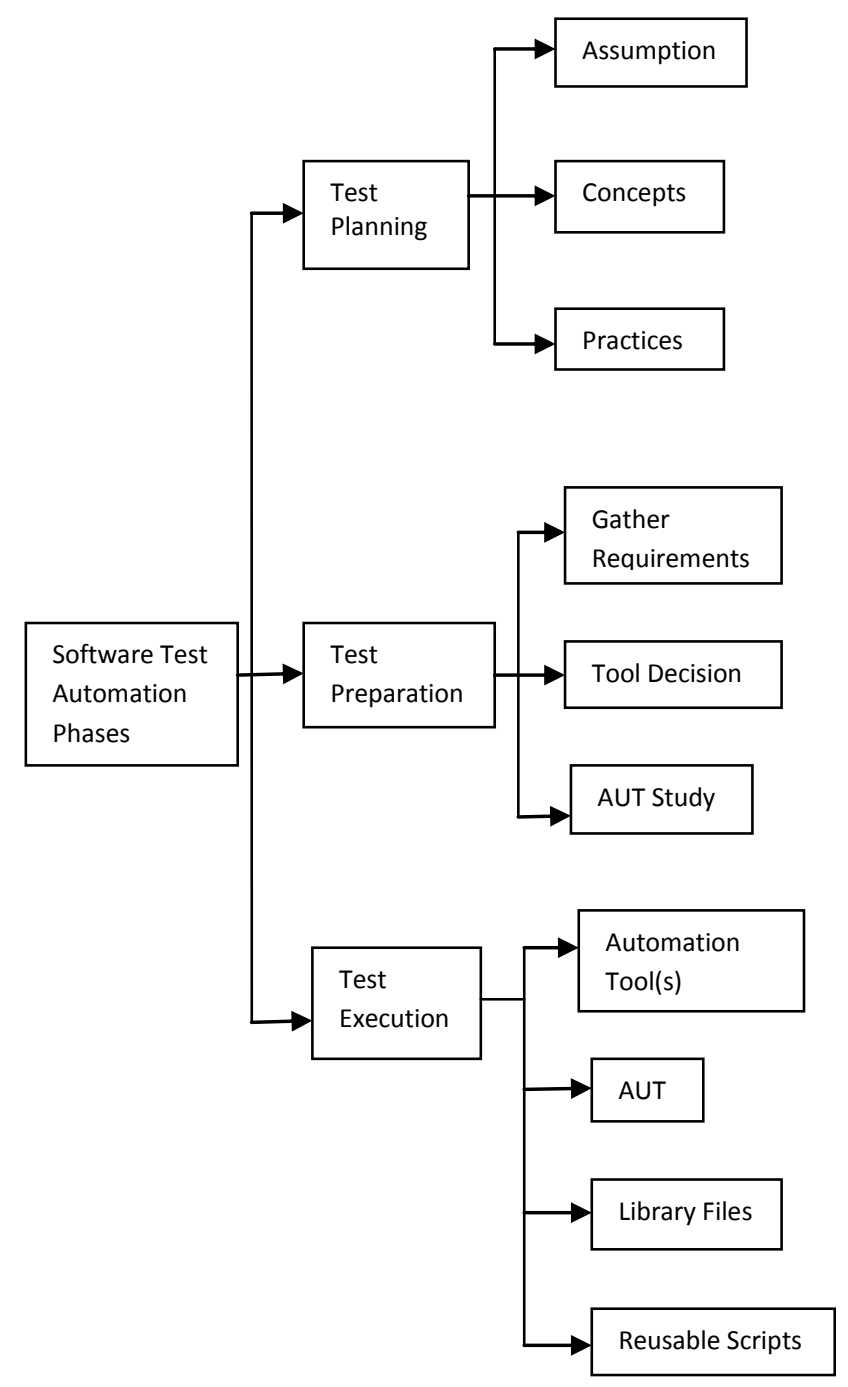

Fig. 1 STAF Phases

The outcome of the phase is the master test plan. Test preparation phase includes activities such as gathering the functional requirements and translating them into test requirement, writing the test cases, studying the application under test (AUT). Performing feasibility analysis with the various automation tools and selecting the appropriate tools. The third and last phase of test automation is Test execution. In this phase, the automated test scripts and library files are implemented and executed against the AUT to generate test report and problems or defects reports. 
STAF is a set of concepts and assumptions that provide support for automated software testing. STAF includes the following for capturing and controlling the following, i.e., test objects, library files, and reusable scripts etc. STAFG are designed and implemented to get the following benefits:

1. STAF ensures that the same library files or methods would be used across the test scripts.

2. STAF provides greater consistency in the entire testing process, i.e., test scripts, test case design, and test flow.

3. STAF reduce amount of code to develop and maintain. A method is written in one library files which are used by the different user. Therefore, there is no need for software tester to write his or her code.

4. STAF maximize reusability and shield non technical testers.

In 2012, Jain and Sharma proposed an efficient keyword driven test automation framework for web application. Keyword driven framework reduces the complex script programming knowledge problem for the automation testing [4]. In [12], Patwa provides an overview of how the hybrid test automation framework can be implemented using QTP. In a technical report of IBM global business services, Rashmi Mascarenhas [13] developed and implemented an automation framework. In 2011, Shahamiri et al. proposed an automated framework for software test oracle. Test Oracles are used as a complete and reliable source of expected outputs [15]. In literature, we have identified different types of automation framework like modular framework (MF), data driven framework (DDF), keyword driven framework (KDF), and hybrid framework (HF); and selecting one of them is not an easy task according to the requirements of software testing process because each technique pursues a specific objective or goal. Therefore, in order to address this issue, we present a method for the selection of software testing automation framework (STAF) using Analytic Hierarchy Process (AHP) by considering the following criteria, i.e., Master Test Script (MTS), Reading of Data from Data Files (RDDF), Data Tables and Keyword (DTK), and Scripts, Data Table and Keywords (SDTK). In [21], we proposed a fuzzy based approach for the selection of Software Testing Automation Framework.

In literature, there are various multi-criteria decision making methods (MCDM) [1, 14, 19] like Multi-Attribute Utility Theory (MAUT), Analytic Hierarchy Process (AHP), Fuzzy Set Theory, Case Based Reasoning, Data Envelopment Analysis, Simple Multiple Attribute Rating Techniques (SMART), Goal Programming, Elimination and Choice Translating Reality (ELECTRE), and PROMETHEE which are used for the selection and prioritization of attributes on the basis of different criteria. There are various applications of these methods in the following area: water management, energy management, engineering economics etc. In this paper, we adopt AHP for the selection of STAF because it is easy to use; and its hierarchical structure can easily adjust to fit many sized problems. In literature, several researchers advocate the use of AHP. For example, in 2014, Khan et al. proposed a method for the selection of software development life cycles using AHP [10]. In our previous work, we present a method for the prioritization of requirements in goal oriented requirements elicitation process using fuzzy extended AHP $[6,7]$.

The paper is organized as follows: In section II, we present different types of STAF like Modular Framework (MF), DataDriven Framework (DDF), Keyword Driven Framework (KDF), and Hybrid Framework (HF). Brief introduction about AHP is given in section III. We present the proposed method for the selection of STAF in section IV. Case study is given in section V. Finally, we conclude the paper in section VI.

\section{SOFTWARE TEST AUTOMATION FRAMEWORK (STAF)}

In this section we present the brief description about the following STAF $[2,5,11,13,20]$.

(a) Modular Framework

(i) Test script modular framework

(ii) Test library modular framework

(b) Data-Driven Framework

(c) Keyword Driven Framework

(d) Hybrid Framework

\section{(a) Modular Framework}

Modular Framework (MF) is built on the concept of abstraction. This involves the creation of independent scripts that represent the modules of AUT. These modules in turn are used in a hierarchical fashion to build large test; where as in library modular framework it creates the library files instead of small, independent scripts. It divides the AUT into functions or procedures that are implemented by library files and called from master scripts. This type of framework will yield higher degree of modularity and overall maintainability of test scripts.

\section{(b) Data-Driven Framework}

In Data- Driven Framework (DDF) input data is read from the data files; and output data is also written in output files. In DDF test script is used repeatedly with varying input and response data that comes from a predefined data set. It reduces coding for large test cases.

\section{(c) Keyword Driven Framework}

In Keyword Driven Framework (KDF), testing is driven by data table and keywords. These data table and keywords are independent from automation tool. Keyword represents the actions which have to perform. The keywords are fed to a driver file which converts the keywords into actions. In addition, functionality of AUT is documented in table format. It's more complex framework than data driven framework. Test cases get longer and require large efforts. KDF needs technical experts to develop the solution.

\section{(d) Hybrid Framework}

This is the combination of all of the above mentioned frameworks. This brings in benefits delivered from all frameworks and trying to mitigate their weaknesses. This type of framework provides the following solution for automation testing: (i) Enhance efficiency during the design and development of automated test scripts, (ii) Time saving, (iii) Built robust and secure framework, (iv) Shield non-technical testers from code. It's more complex framework and it requires large efforts and maintainability. Increase dependency on technical experts to develop the solution.

\section{ANALYTIC HIERARCHY PROCESS}

In 1972, T. L. Saaty proposed the analytic hierarchy process [14]. It is a multi-criteria decision (MCDM) making method AHP helps decision maker facing a complex problem with multiple conflicting and subjective criteria [1, 3, 14]. This process permits the hierarchical structure of the criteria or subcriteria when allocating a weight. AHP involves following steps: (a) problem definition (b) pair-wise comparisons (c) compute the eigenvector of the relative importance of the criteria (d) check 
consistency. Once we have identified the criteria or sub-criteria according to the need of the problem or problem definition, then the next step is to express the decision makers opinion on only two alternatives than simultaneously on all the alternatives. On the basis of the pair wise comparison with all the alternatives, we construct the pair-wise comparison matrix on the basis of the following rating scale (Judgment scale)

Table 1: The Saaty rating scale

\begin{tabular}{|c|l|}
\hline $\begin{array}{l}\text { Intensity of } \\
\text { importance }\end{array}$ & Definition \\
\hline 1 & Equal importance \\
\hline 3 & Somewhat more importance \\
\hline 5 & Much more important \\
\hline 7 & Very much important \\
\hline 9 & Absolutely more important \\
\hline $2,4,6,8$ & $\begin{array}{l}\text { Intermediates values ( when } \\
\text { compromise is needed) }\end{array}$ \\
\hline
\end{tabular}

There are several methods or algorithm for the calculation of eigenvector. In this paper, we adopt the following algorithm [10]:

\section{Algorithm:}

Step 1: Multiplying together the entries in each row of the matrix and then take the $\mathrm{n}^{\text {th }}$ root of the product.

Step 2: Compute the sum of $\mathrm{n}^{\text {th }}$ root and store the result in SUM. Step 3: The value of SUM would be used to normalize the product values and the resultant would be the eigenvector

Saaty argues that a Consistency Ratio $(\mathrm{CR})>0.1$ indicates that the judgment are at the limit of consistency, where as $\mathrm{CR}=0.9$ would mean that the pair wise judgment are random and are completely untrustworthy $[1,14]$.

\section{PROPOSED METHOD}

This section presents a method for the selection of STAF using AHP. The proposed method is presented simply in the following:

(i) Identify the criteria for the selection of STAF

(ii) Construct the hierarchical structure of STAF

(iii) Construct the decision matrix

(iv) Calculate the ranking values

(v) Selection of STAF

\section{(i) Identify the criteria}

Before the selection of any STAF, software tester should identify the criteria's for the selection of STAF. On the basis of our literature review, we have identified the following criteria which influence the decision of choosing a STAF:

(a) Master Test Script (MTS),

(b) Reading of Data from Data Files (RDDF)

(c) Data Tables and Keywords (DTK), and

(d) Scripts, Data Tables and Keywords (SDTK)

\section{(ii) Construct the hierarchical structure of}

\section{STAF}

As the STAF selection decision requires a systematic approach to help integrate different attributes or criteria into software testing development. Therefore, it is essential to break down the problem into more manageable sub-problems. As illustrated in Fig.2, the problem studied here has three level of hierarchy. The first level, i.e., the overall objective, is the selection of a STAF. Level two contains four different STAF like MF, DDF, KDF, and HF; and at level three following decision criteria is given Master Test Script, Reading of Data from Data Files, Data Tables and Keywords, and Script, Data Tables and Keywords.

\section{(iii) Construct the Decision Matrix}

We will create the decision matrix using AHP method $[1,8,14]$. Detailed description for the construction of decision matrix is given in section $\mathrm{V}$.

\section{(iv) Calculate the Ranking Values}

Ranking values will be obtained after computing the eigenvector values from the pair wise comparison matrix $[1,8,14]$.

\section{(v) Selection of STAF}

Construct the binary search tree of the ranking values (BSRTV) that we have obtained in previous step. Apply in-order tree traversal technique on BSTRV and as a result, we will get the prioritized list of STAF. The model which has highest priority will be selected for the testing of the project.

\section{CASE STUDY}

This section presents a case study of our work. In-order to test any software, it is indispensible to select STAF according to the need of our project. There are various STAF which are available in the literature. In this paper, we have considered the project, entitled "Online Shopping". We have identified the following criteria for the selection of STAF: Master Test Script (MTS), Reading of Data from Data Files (RDDF), Data Tables and Keyword (DTK), and Scripts, Data Table and Keywords (SDTK). The hierarchical structure of the STAF selection problem is given in Fig. 2 (Step second). For the third step, we have defined the initial matrix for the pair wise comparison. In this matrix, the principal diagonal matrix contains entries of 1 because each factor is important as itself.

Table 2: Initial Matrix

\begin{tabular}{|l|l|l|l|l|}
\hline Criteria & MTS & RDDF & DTK & SDTK \\
\hline MTS & 1 & & & \\
\hline RDDF & & 1 & & \\
\hline DTK & & & 1 & \\
\hline SDTK & & & & 1 \\
\hline
\end{tabular}

To make the pair wise comparison among all the criteria, we decide that RDDF is more important than MTS. In the next matrix, i.e., Table 3, that is rated as 3 in the cell RDDF and MTS; and 1/3in MTS and RDDF. We also decide that SDTK is more important than MTS. Therefore, in Table 4, we put 7 in SDTK and MTS; and 1/7 in MTS and SDTK. In a similar way, we complete the matrix, that we call the "Overall Preference Matrix (OPM)". 


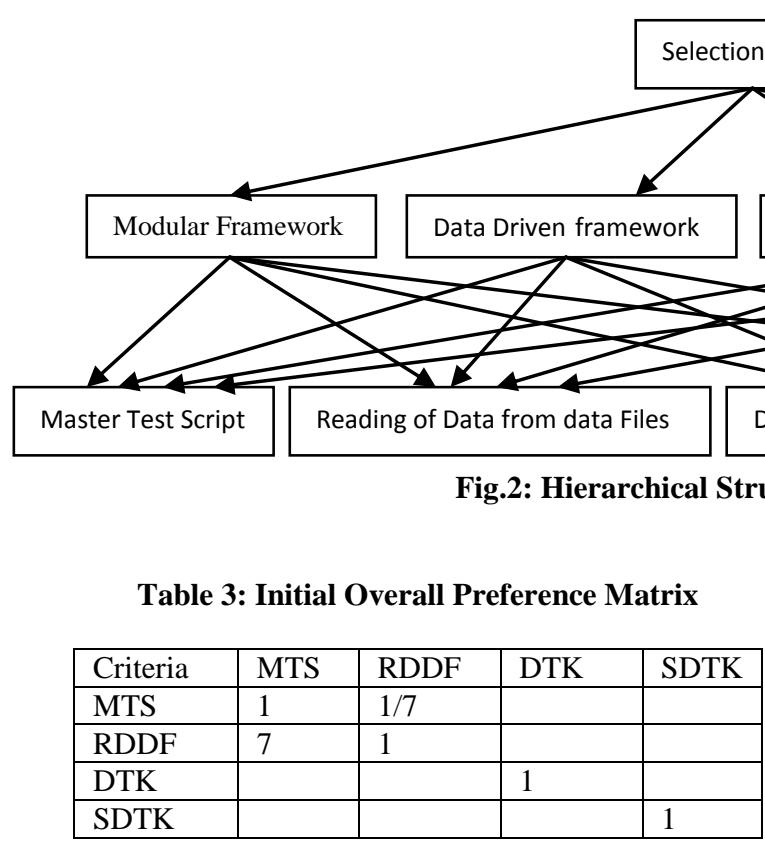

Table 4: Overall Preference Matrix

\begin{tabular}{|l|l|l|l|l|}
\hline Criteria & MTS & RDDF & DTK & SDTK \\
\hline MTS & 1 & $1 / 3$ & $1 / 5$ & $1 / 7$ \\
\hline RDDF & 3 & 1 & $1 / 5$ & $1 / 3$ \\
\hline DTK & 5 & 5 & 1 & $1 / 5$ \\
\hline SDTK & 7 & 3 & 5 & 1 \\
\hline
\end{tabular}

The eigenvector or relative value vector (RVV) corresponding to each criterion is calculated by the algorithm, given in section III. Therefore, as a result, we have identified the following values: $(0.312,0.669,1.4953,3.201)$. These four values correspond to the relative value of MTS, RDDF, DTK, and SDTK. The value 3.201 means that SDTK is an important criterion. 1.4953 shows that DTK is also an important parameter for the selection of STAF. The remaining two data represent that MTS and RDDF are least considerable parameters.

After this, we evaluate different STAF on the basis of the given parameters, i.e., MTS, RDDF, DTK, and SDTK. Table 5, Table 6, Table 7, and Table 8 are created according to the pairwise comparisons w. r. t. MTS, RDDF, DTK, and SDTK respectively.

Table 5: Pair-wise comparison matrix w. r. t. MTS

\begin{tabular}{|l|l|l|l|l|l|}
\hline Criteria & MF & DDF & KDF & HF & $\begin{array}{l}\text { Priority } \\
\text { Vector }\end{array}$ \\
\hline MF & 1 & 5 & 5 & $1 / 7$ & 1.374 \\
\hline DDF & $1 / 5$ & 1 & 1 & $1 / 5$ & 0.447 \\
\hline KDF & $1 / 5$ & 1 & 1 & $1 / 7$ & 0.411 \\
\hline HF & 7 & 5 & 7 & 1 & 3.96 \\
\hline
\end{tabular}

Table 6: Pair-wise comparison matrix w. r. t. RDDF

\begin{tabular}{|l|l|l|l|l|l|}
\hline Criteria & MF & DDF & KDF & HF & $\begin{array}{l}\text { Priority } \\
\text { Vector }\end{array}$ \\
\hline MF & 1 & $1 / 5$ & 1 & $1 / 7$ & 0.411 \\
\hline DDF & 5 & 1 & 7 & $1 / 5$ & 1.626 \\
\hline KDF & 1 & $1 / 7$ & 1 & $1 / 3$ & 0.467 \\
\hline HF & 7 & 5 & 3 & 1 & 3.201 \\
\hline
\end{tabular}

Table 7: Pair-wise comparison matrix w. r. t. DTK

\begin{tabular}{|l|l|l|l|l|l|}
\hline Criteria & MF & DDF & KDF & HF & $\begin{array}{l}\text { Priority } \\
\text { Vector }\end{array}$ \\
\hline MF & 1 & 1 & $1 / 5$ & $1 / 7$ & 0.411 \\
\hline DDF & 1 & 1 & $1 / 5$ & $1 / 5$ & 0.447 \\
\hline KDF & 5 & 5 & 1 & $1 / 3$ & 1.699 \\
\hline HF & 7 & 5 & $1 / 3$ & 1 & 1.848 \\
\hline
\end{tabular}

Table 5: Pair-wise comparison matrix w. r. t. SDTK

\begin{tabular}{|l|l|l|l|l|l|}
\hline Criteria & MF & DDF & KDF & HF & $\begin{array}{l}\text { Priority } \\
\text { Vector }\end{array}$ \\
\hline MF & 1 & 1 & 1 & $1 / 9$ & 0.577 \\
\hline DDF & 1 & 1 & 1 & $1 / 9$ & 0.577 \\
\hline KDF & 1 & 1 & 1 & $1 / 9$ & 0.577 \\
\hline HF & 9 & 9 & 9 & 1 & 5.196 \\
\hline
\end{tabular}

Table 9: Final Decision Matrix

\begin{tabular}{|c|c|c|c|c|c|}
\hline \multirow{3}{*}{ STT } & \multicolumn{4}{|c|}{ Criteria } & \multirow{3}{*}{$\begin{array}{l}\text { Priority } \\
\text { Vector }\end{array}$} \\
\hline & MF & DDF & KDF & $\mathrm{HF}$ & \\
\hline & 0.312 & 0.669 & 1.495 & 3.201 & \\
\hline MF & 1.374 & 0.411 & 0.411 & 0.577 & 3.165 \\
\hline DDF & 0.447 & 1.626 & 0.447 & 0.577 & 3.742 \\
\hline KDF & 0.411 & 0.467 & 1.699 & 0.577 & 4.827 \\
\hline $\mathrm{HF}$ & 3.96 & 3.201 & 1.848 & 5.196 & 22.77 \\
\hline
\end{tabular}

On the basis of results given in Table 9, we select the hybrid framework (HF) because the value of the priority vector of HF is 22.77 .

\section{CONCLUSION}

This paper presents a method for the selection of STAF using AHP. Proposed method is a five step process, namely, (i) identify the criteria for the selection of SATF, (ii) construct the hierarchical structure of STAF, (iii) construct the decision matrix, (iv) calculate the ranking values, and (v) the selection of a STAF. In this paper, we have considered four criteria's for the selection of STAF, i.e., MTS, RDDF, DTK, and SDTK; and as a result we select hybrid Framework (HF) for the testing of Online Shopping System (OSS). Future research agenda includes the following:

1. To propose a fuzzy decision making approach for the selection of STAF.

2. To propose a method for the selection of STAF using hybrid techniques like fuzzy AHP and fuzzy ANP. 


\section{REFERENCES}

[1] A. Ishizaka and A. Labib, "Review of the Main Development in the Analytic Hierarchy Process", Experts Systems with Applications, Vol.38, No.11, pp. 1433614345, 2011.

[2] Divya A, Mahalakshmi S D, “ An Efficient Framework for Unified Automation Testing: A Case Study on Software Industry", International Journal of Advanced Research in Computer Science \& Technology, Vol. 2, Issue Special 1, Jan-March 2014,

[3] Ishizaka and A. Labib, "Review of the Main Development in the Analytic Hierarchy Process", Experts Systems with Applications, Vol.38, No.11, pp. 14336-14345, 2011.

[4] Jain and Sharma, "An Efficient Keyword Driven Test Automation Framework for Web Applications", International Journal of Engineering Science and Advanced Technology, Volume-2, Issue-3, 2012

[5] Laukkanen Pekka, "Data-Driven and Keyword-Driven Test Automation Frameworks", M. Tech Thesis, Department of Computer Science and Engineering Software Business and Engineering Institute, Helsinki University of Technology, 2006.

[6] Mohd Sadiq and S K Jain, "Applying Fuzzy Preference Relation for Requirements Prioritization in Goal Oriented Requirements Elicitation Process", International Journal of Systems Assurance Engineering and Maintenance, Springer, March 2014, ISSN No.: 0975-6809 (Print Version) ISSN No.: 0976-4348 (Electronic Version).

[7] Mohd Sadiq, S K Jain, “ A Fuzzy Based Approach for Requirements Prioritization in Goal Oriented Requirements Elicitation Process", 25th International Conference on Software Engineering and Knowledge Engineering, Boston, USA, June 27-June 29, 2013

[8] Mohd. Sadiq, Shabina Ghafir, Mohd. Shahid, "An Approach for Eliciting Software Requirements and its Prioritization using Analytic Hierarchy Process", IEEE International Conference on Advances in Recent Technologies in Communication and Computing, pp.790795 Kerala, India, 2009.

[9] Mumtaz Ahmad Khan and Mohd. Sadiq, "Analysis of Black Box Software Testing Techniques: A Case Study",
IEEE International Conference and Workshop on Current Trends in Information Technology, pp.1-5, December, 2011, Dubai, UAE.

[10] Mumtaz Ahmed Khan, Azra Parveen, and Mohd. Sadiq, "A Method for the Selection of Software Development Life Cycle Models Using Analytic Hierarchy Process", IEEE International Conference on Issues and Challenges in Intelligent Computing Techniques (ICICT), pp. 539-545, 2014, KIET Ghaziabad, UP, India.

[11] Pajunen T, Takala T, Katara M, "Model based testing with a General Purpose Keyword-Driven Test Automation framework", Software testing, Verification and validation Workshop, pp. 242-251, 2011

[12] Patwa P, "Hybrid Test Automation Frameworks Implementation using QTP”, Technical Article

[13] Rashmi Mascarenhas, "Developing and Implementing an Automation Framework", IBM Global Business Services, 2008

[14] Saaty T, "The Analytic Hierarchy Process", New York: McGraw-Hill, 1980.

[15] Shahamiri et al, "An Automated Framework for Software Test Oracle", Information and Software Technology, Elsevier, 2011

[16] Sommerville, "Software Engineering", Fifth Edition, New York: Addison Wesley, 1996.

[17] The Standish Group International, CHAOS Summary 2009.

[18] Trivedi S H, "Software Testing Techniques", Internationa Journal of Advanced Research in Computer Science and Software Engineering, Volume 2, Issue 10, October 2012

[19] Velasquez M and Hester P T, "An Analysis of Multicriteria Decision making Methods", International Journal of Operations research”, Vol. 10, No.2, pp. 56-66, 2013

[20] Viera et al., "Automation of GUI Testing Using Model Driven Approach", ACM- AST, China, 2006.

[21] Mohd Sadiq and Fahamida Firoze, "A Fuzzy Based Approach for the Selection of Software Testing Automation Framework", International Conference on Computational Intelligence in Data Mining, Springer, 2014. 